\title{
Improvement of Physical Layer Security Performance of Wireless Relay Communication System
}

\author{
Bao-Feng Yang, You-Fan Zhu, Yuan-Yuan Gao \\ Institution of Communications Engineering, PLA University of Science and Technology, Nanjing, 210007, China
}

\author{
Xiao-Bing Zhang \\ Nanjing University of Science and Technology, Nanjing, \\ 210001, China \\ E-mail: my770809@tom.com
}

\author{
Xin-Ling Lu \\ PLA 69296 Unit, Kashi, 844200, \\ E-mail: my770809@tom.com
}

\begin{abstract}
Due to the broadcast nature of wireless communications, security performance of conventional wireless relay communication system is difficult to be guaranteed. In this paper, security performance of the wireless relay communication system based on physical layer network coding (PLNC) is studied. The theoretical analyses and simulations show that the physical layer security performance of wireless relay communication system can be improved effectively by using PLNC technique. Moreover, this paper also shows that the location of relay node in the network has a big impact on the security performance of system.
\end{abstract}

Keywords-physical layer security; network coding; security capacity; wireless relay communication system

\section{INTRODUCTION}

Wireless relay communication system can reduce costs, expand the coverage of communications, and increase the transmission rate of the mobile communication systems with relay technology. However, due to the broadcast nature and openness of electromagnetic signals, the enemy's passive eavesdropping becomes a major threat to the security of the wireless relay communication system.

In 1975, Wyner ${ }^{[1]}$ proposed the basic model of physical layer security - tapping channel model, and proved that when capacity of tapping channel was inferior to capacity of the main channel, any eavesdropper could not obtain the information. At this time, the rate of information between the source and the destination was defined as security information rate, and the maximum rate of security information rate is called security capacity (Cs). When information rate was not greater than Cs, communication security could be guaranteed and any eavesdropper could not get any useful information from the received message.

In 2006, Zhang Shengli creatively put forward PLNC technology ${ }^{[2]}$. The traditional relay system aimed to avoid interference with each other, and it required four slots. NC(Network layer) Network Coding was proposed to reduce 4 slots to 3 slots required by a two-way communication. PLNC was proposed to solve the problem of signal interference, which reduced 3 slots to 2 slots by using a two-way communication. This method was applied to make the eavesdropper subject to interference, so that interference of eavesdropper was much greater than that of receiver, then improving the security performance of physical layer.
Based on Wyner's eavesdropping channel model, this paper studies security performance of wireless relay communication system based on physical layer network coding when the wiretap channel information is unknown. Moreover, this paper also studies the impact of relay node location on physical layer security performance of the system by regarding the security zone as a measure indicator.

\section{THE SECURITY PERFORMANCE OF} WIRELESS RELAY COMMUNICATION SYSTEM

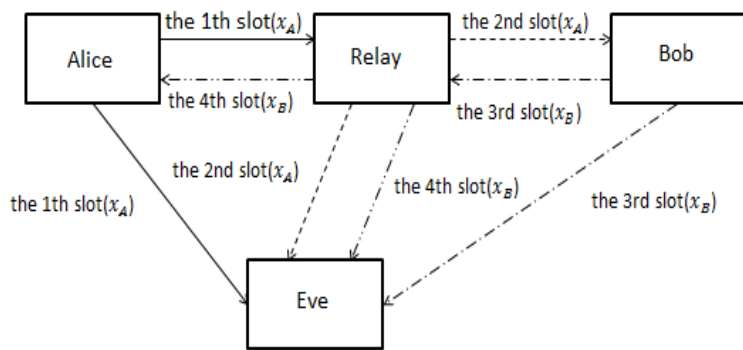

Figure 1. Traditional relay transmission mechanism with eavesdropper.

We suppose the wireless relay communication system has three nodes ${ }^{[4]}$, and valid communication sides are Alice and Bob respectively, which is shown in Fig.1. Relay adopts amplify-and-forward strategies. In this model, a passive eavesdropper Eve is also considered. Eve's location is unknown. Here we suppose Alice is denoted as A, Bob is denoted as B, Eve as E, Relay as R. Assuming that loss factor $\alpha(0<\alpha<1)$ in Additive White Gaussian Noise (AWGN) channel path and communication is inversely proportional to the square of the distance on both sides. Loss factor of AR, $\mathrm{BR}, \mathrm{AE}, \mathrm{BE}$ and $\mathrm{RE}$ is $\alpha_{\mathrm{A}}, \alpha_{\mathrm{B}}, \alpha_{1}, \alpha_{2}, \alpha_{3}$. Amplification coefficient of $\mathrm{R}$ is $1 / \alpha_{\mathrm{A}} . e_{\mathrm{A}}^{n}, e_{\mathrm{B}}^{n}, e_{1}^{n}, e_{2}^{n}$ and $e_{3}^{n}$ represent the AWGN of $\mathrm{AR}, \mathrm{BR}, \mathrm{AE}, \mathrm{BE}$ and $\mathrm{RE}$ in the $\mathrm{n}$ slot respectively, and $e_{A}^{n} \sim\left(0, \sigma_{A}^{2}\right), e_{B}^{n} \sim\left(0, \sigma_{B}^{2}\right)$, $e_{1}^{n} \sim\left(0, \sigma_{1}^{2}\right), e_{2}^{n} \sim\left(0, \sigma_{2}^{2}\right), e_{3}^{n} \sim\left(0, \sigma_{3}^{2}\right)$. Given that $\mathrm{A}$ sends a signal of to $\mathrm{B}$ is $x_{\mathrm{A}}$, and $\mathrm{B}$ will send a signal to $\mathrm{A}$ is $x_{\mathrm{B}}$, $x_{A}^{n}$ represents the $\mathrm{n}$-th symbol sent by $\mathrm{A}$, and $x_{B}^{n}$ represents the n-th symbol sent by $B$. 
According to Shannon formula ${ }^{[5]}$, the channel capacity from $B$ to $A\left(C_{B A}\right)$, from $A$ to $B\left(C_{A B}\right)$, from $A$ to $E\left(C_{A E}\right)$ and the channel capacity of $\mathrm{A}\left(\mathrm{C}_{\mathrm{BE}}\right)$ is:

$$
\begin{gathered}
\mathrm{C}_{\mathrm{BA}}=\frac{1}{2} \log _{2}\left(1+\frac{\alpha_{\mathrm{B}}^{2} * \mathrm{P}}{\sigma_{\mathrm{A}}^{2}+\sigma_{\mathrm{B}}^{2}}\right) \\
\mathrm{C}_{\mathrm{AB}}=\frac{1}{2} \log _{2}\left(1+\frac{\alpha_{\mathrm{B}}^{2} * \mathrm{P}}{\left(\alpha_{\mathrm{B}} / \alpha_{\mathrm{A}}\right)^{2} \sigma_{\mathrm{A}}^{2}+\sigma_{\mathrm{B}}^{2}}\right) \\
\mathrm{C}_{\mathrm{AE}}=\frac{1}{2} \log _{2}\left(1+\max \left[\frac{\alpha_{3}^{2} * \mathrm{P}}{\left(\alpha_{3} / \alpha_{\mathrm{A}}\right)^{2} \sigma_{\mathrm{A}}^{2}+\sigma_{3}^{2}}, \frac{\alpha_{1}^{2} * \mathrm{P}}{\sigma_{1}^{2}}\right]\right. \\
\mathrm{C}_{\mathrm{BE}}=\frac{1}{2} \log _{2}\left(1+\max \left[\frac{\left(\alpha_{3} \alpha_{\mathrm{B}} / \alpha_{\mathrm{A}}\right)^{2} * \mathrm{P}}{\left(\alpha_{3} / \alpha_{\mathrm{A}}\right)^{2} \sigma_{\mathrm{B}}^{2}+\sigma_{3}^{2}}, \frac{\alpha_{2}^{2} * \mathrm{P}}{\sigma_{2}^{2}}\right]\right) \\
\mathrm{C}_{\mathrm{SA}}=\mathrm{C}_{\mathrm{BA}}-\mathrm{C}_{\mathrm{BE}}=\frac{1}{2} \log _{2}\left(1+\frac{\alpha_{\mathrm{B}}^{2} * \mathrm{P}}{\sigma_{\mathrm{A}}^{2}+\sigma^{2}}\right) \\
\mathrm{C}_{\mathrm{SB}}=\mathrm{C}_{\mathrm{AB}}-\mathrm{C}_{\mathrm{AE}}=\frac{1}{2} \log _{2}\left(1+\frac{\alpha_{\mathrm{B}}^{2} * \mathrm{P}}{\left(\alpha_{\mathrm{B}} / \alpha_{\mathrm{A}}\right)^{2} \sigma_{\mathrm{A}}^{2}}\right. \\
\text { III. STUDY ON PHYSICAL LAYER SECURITY } \\
\text { PERFORMANCE OF WIRELESS RELAY } \\
\text { COMMUNICATION SYSTEM BASED ON PLNC }
\end{gathered}
$$

\section{STUDY ON PHYSICAL LAYER SECURITY PERFORMANCE OF WIRELESS RELAY COMMUNICATION SYSTEM BASED ON PLNC}$$
\mathrm{C}_{\mathrm{SA}}=\mathrm{C}_{\mathrm{BA}}-\mathrm{C}_{\mathrm{BE}}=\frac{1}{2} \log _{2}\left(1+\frac{\alpha_{\mathrm{B}}^{2} * \mathrm{P}}{\sigma_{\mathrm{A}}^{2}+\sigma_{\mathrm{B}}^{2}}\right)-\frac{1}{2} \log _{2}\left(1+\max \left[\frac{\left(\alpha_{3} \alpha_{\mathrm{B}} / \alpha_{\mathrm{A}}\right)^{2} * \mathrm{P}}{\left(\alpha_{3} / \alpha_{\mathrm{A}}\right)^{2} \sigma_{\mathrm{B}}^{2}+\sigma_{3}^{2}}, \frac{\alpha_{2}^{2} * \mathrm{P}}{\sigma_{2}^{2}}\right]\right)
$$

$$
\mathrm{C}_{\mathrm{SB}}=\mathrm{C}_{\mathrm{AB}}-\mathrm{C}_{\mathrm{AE}}=\frac{1}{2} \log _{2}\left(1+\frac{\alpha_{\mathrm{B}}^{2} * \mathrm{P}}{\left(\alpha_{\mathrm{B}} / \alpha_{\mathrm{A}}\right)^{2} \sigma_{\mathrm{A}}^{2}+\sigma_{\mathrm{B}}^{2}}\right)-\frac{1}{2} \log _{2}\left(1+\max \left[\frac{\alpha_{3}^{2} * \mathrm{P}}{\left(\alpha_{3} / \alpha_{\mathrm{A}}\right)^{2} \sigma_{\mathrm{A}}^{2}+\sigma_{3}^{2}}, \frac{\alpha_{1}^{2} * \mathrm{P}}{\sigma_{1}^{2}}\right]\right)
$$

Wyner has proved that ${ }^{[6]}$ : As long as hacking channel capacity is inferior to that of the main channel $\left(\mathrm{C}_{\mathrm{w}}<\mathrm{C}_{\mathrm{M}}\right.$, Cs $>$ 0 ), theoretically there must be some kind of communication scheme that can ensure communication security. When the system information transmission rate satisfies $\mathrm{R}<=\mathrm{Cs}$, the legitimate communication node can achieve secure communication. So the security capacity is defined as the difference between the main channel capacity $C_{M}$ and wiretap channel capacity $C_{W}$, namely, $C s=\left[C_{M}-C_{W}\right]$.

Therefore, security capacity of A can be expressed as:

Similarly, security capacity of B can be expressed as:

The communication process of the wireless relay system based on PLNC is shown as Fig.2.

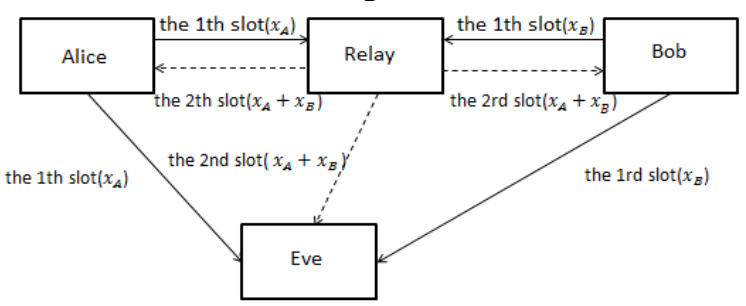

Figure 2. PLNC transport mechanism with eavesdropper.

$$
\begin{gathered}
\mathrm{C}_{\mathrm{AE}}=\frac{1}{2} \log _{2}\left(1+\max \left[\frac{\alpha_{3}^{2} * \mathrm{P}}{\left(\alpha_{\mathrm{B}} \alpha_{3} / \alpha_{\mathrm{A}}\right)^{2} * \mathrm{P}+\left(\alpha_{3} / \alpha_{\mathrm{A}}\right)^{2} \sigma_{\mathrm{A}}^{2}+\left(\alpha_{3} / \alpha_{\mathrm{A}}\right)^{2} \sigma_{\mathrm{B}}^{2}+\sigma_{3}^{2}}, \frac{\alpha_{1}{ }^{2} * \mathrm{P}}{\alpha_{2}{ }^{2} * \mathrm{P}+\sigma_{1}^{2}+\sigma_{1}^{2}}\right]\right) \\
\mathrm{C}_{\mathrm{BE}}=\frac{1}{2} \log _{2}\left(1+\max \left[\frac{\left(\alpha_{\mathrm{B}} \alpha_{3} / \alpha_{\mathrm{A}}\right)^{2} * \mathrm{P}}{\alpha_{3}{ }^{2} * \mathrm{P}+\left(\alpha_{3} / \alpha_{\mathrm{A}}\right)^{2} \sigma_{\mathrm{A}}^{2}+\left(\alpha_{3} / \alpha_{\mathrm{A}}\right)^{2} \sigma_{\mathrm{B}}^{2}+\sigma_{3}^{2}}, \frac{\alpha^{2}{ }^{2} * \mathrm{P}}{\alpha_{1}^{2} * \mathrm{P}+\sigma_{1}^{2}+\sigma_{1}^{2}}\right]\right)
\end{gathered}
$$

So the security capacity of A is:

$$
\mathrm{C}_{\mathrm{SA}}=\mathrm{C}_{\mathrm{BA}}-\mathrm{C}_{\mathrm{BE}}=\frac{1}{2} \log _{2}\left(1+\frac{\alpha_{\mathrm{B}}^{2} * \mathrm{P}}{2 \sigma_{\mathrm{A}}^{2}+\sigma_{\mathrm{B}}^{2}}\right)-\frac{1}{2} \log _{2}(1+\mathrm{N})
$$


Where

$$
\mathrm{N}=\max \left[\frac{\left(\alpha_{\mathrm{B}} \alpha_{3} / \alpha_{\mathrm{A}}\right)^{2} * \mathrm{P}}{\alpha_{3}^{2} * \mathrm{P}+\left(\alpha_{3} / \alpha_{\mathrm{A}}\right)^{2} \sigma_{\mathrm{A}}^{2}+\left(\alpha_{3} / \alpha_{\mathrm{A}}\right)^{2}{\sigma_{\mathrm{B}}^{2}+\sigma_{3}^{2}}_{2}}, \frac{\alpha_{2}{ }^{2} * \mathrm{P}}{\alpha_{1}^{2} * \mathrm{P}+\sigma_{1}^{2}+\sigma_{1}^{2}}\right]
$$

Similarly, the security capacity of $\mathrm{B}$ is:

$$
\begin{gathered}
\mathrm{C}_{\mathrm{SB}}=\mathrm{C}_{\mathrm{AB}}-\mathrm{C}_{\mathrm{AE}}=\frac{1}{2} \log _{2}\left(1+\frac{\alpha_{\mathrm{B}}{ }^{2} * \mathrm{P}}{\left(\alpha_{\mathrm{B}} / \alpha_{\mathrm{A}}\right)^{2} \sigma_{\mathrm{A}}^{2}+\left[1+\left(\alpha_{\mathrm{B}} / \alpha_{\mathrm{A}}\right)^{2}\right] \sigma_{\mathrm{B}}^{2}}\right)- \\
\frac{1}{2} \log _{2}(1+\mathrm{N})
\end{gathered}
$$

Where

$$
\mathrm{N}=\max \left[\frac{\alpha_{3}{ }^{2} * \mathrm{P}}{\left(\alpha_{\mathrm{B}} \alpha_{3} / \alpha_{\mathrm{A}}\right)^{2} * \mathrm{P}+\left(\alpha_{3} / \alpha_{\mathrm{A}}\right)^{2} \sigma_{\mathrm{A}}^{2}+\left(\alpha_{3} / \alpha_{\mathrm{A}}\right)^{2}{\sigma_{\mathrm{B}}}^{2}+\sigma_{3}^{2}}, \frac{\alpha_{1}{ }^{2} * \mathrm{P}}{\alpha_{2}{ }^{2} * \mathrm{P}+\sigma_{1}^{2}+\sigma_{1}^{2}}\right]
$$

\section{LATIONS AND ANALYSIS}

Assuming the distance between A and B is 20 meters. Given the position of $\mathrm{R}$ is $(10,10), \mathrm{E}$ is any point in the space.

$\mathrm{A}$ and $\mathrm{B}$ are on an article line, in which $\mathrm{A}$ is on origin of coordinates, B's coordinate is $(20,0)$, and R's coordinate is $(10,10)$. The range of $\mathrm{E}$ is $(-20 \leq x \leq 20,-20 \leq y \leq 20)$. When $\mathrm{B}$ is the transmitting terminal, the range of $\mathrm{E}$ is $(0 \leq x \leq 40,-20 \leq$ $y \leq 20)$. According to the analysis of two data sets selected from various parameters in the model:

Low signal to noise ratio: $e_{A}^{n}=2 \times 10^{-4} \mathrm{~W}, e_{B}^{n}=2 \times 10^{-4} \mathrm{~W}$, $e_{1}^{n}=10^{-4} \mathrm{~W}, e_{2}^{n}=10^{-4} \mathrm{~W}$,transmit power of $\mathrm{A}$ and $\mathrm{B}$ reach $10 \mathrm{~W}$.

High signal to noise ratio: $e_{A}^{n}=10^{-4} \mathrm{~W}, e_{B}^{n}=10^{-4} \mathrm{~W}, e_{1}^{n}$ $=10^{-4} \mathrm{~W}, e_{2}^{n}=10^{-4} \mathrm{~W}$,transmit power of $\mathrm{A}$ and $\mathrm{B}$ reach $10 \mathrm{~W}$. (a)

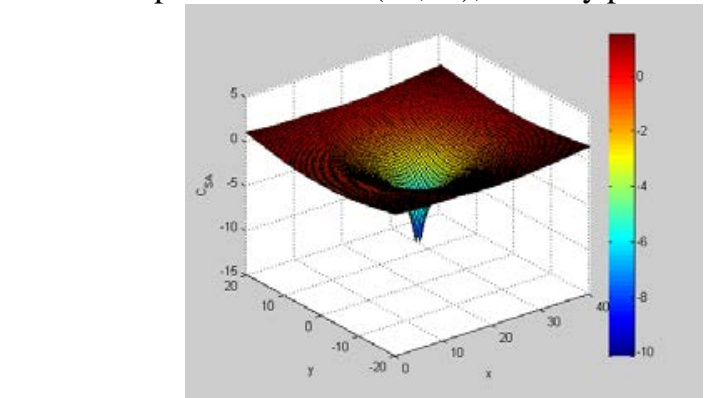

Figure 3 Traditional models with low SNR (a) $\mathrm{C}_{\mathrm{SA}}$; (b) $\mathrm{C}_{\mathrm{SB}}$

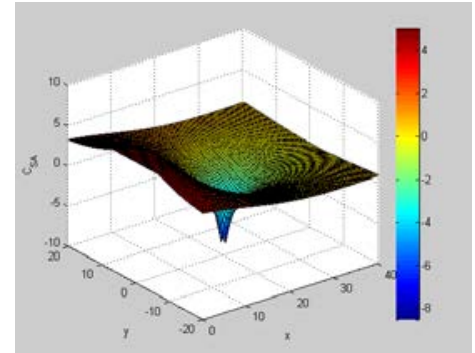

(a)

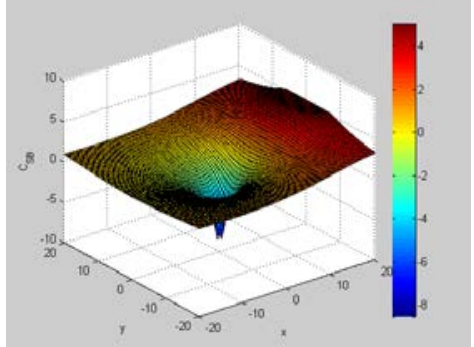

(b)

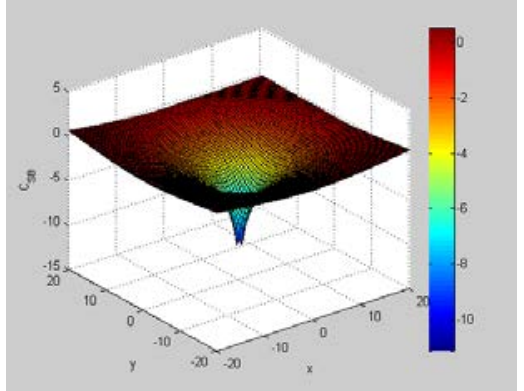

(b)

Figure 4. PLNC model with low SNR (a) $\mathrm{C}_{\mathrm{SA}}$; (b) $\mathrm{C}_{\mathrm{SB}}$; (c) $\mathrm{C}_{\mathrm{SA}}$ (side view).

Fig. 3(a) and (b) show that in traditional communications mode, when eavesdropper gradually leaves away from transmitting terminal, security capacity increases. Fig.4 (a) and (b) show that security capacity has been improved by using PLNC technique. For example, in Fig.3 (a) $x=0$ and $y=20, \mathrm{C}_{\mathrm{s}}=-0.3389 \mathrm{bit} / \mathrm{symbol}$. When PLNC is adopted (in Fig. 4(a)), $x=0, y=20, C_{S}=3.162 \mathrm{bit} / \mathrm{symbol}$. This indicates that PLNC technique greatly enhances the physical security performance of the system, thereby effectively deterring eavesdroppers to access to information. 


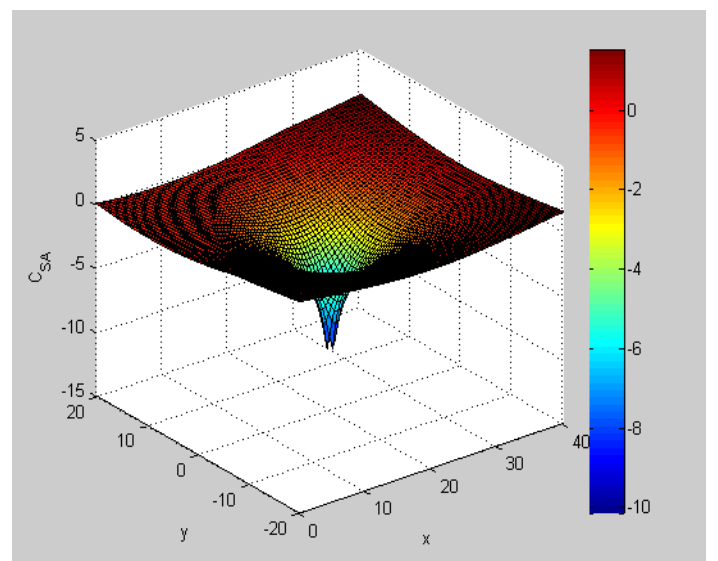

Figure 5. Traditional model $\mathrm{C}_{\mathrm{SA}}$ with high SNR.

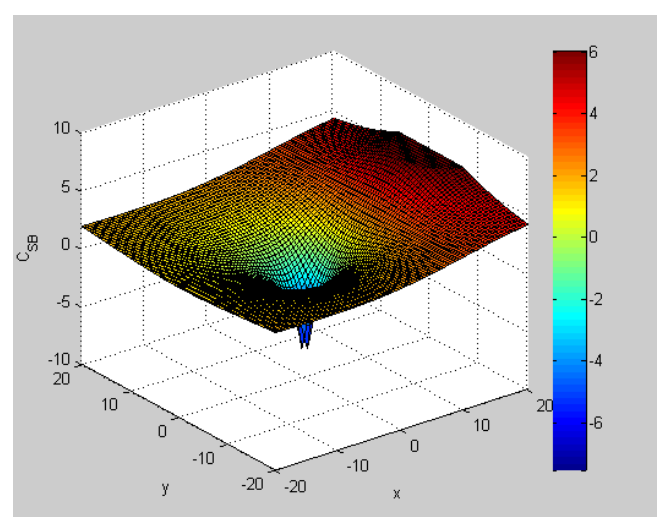

Figure 6. PLNC mode $\mathrm{C}_{\mathrm{SB}}$ with high SNR.

From Fig. 3(a) and Fig. 5, Fig. 4(b) and Fig. 6, it can be drawn, with high signal to noise ratio, security capacity improves but not significantly. For example, in traditional model $\mathrm{C}_{\mathrm{SA}}$ with low SNR $(x=10, y=20)$, security capacity improve is about $-0.178 \mathrm{bit} / \mathrm{symbol}$, while in traditional model $\mathrm{C}_{\mathrm{SA}}$ with high SNR $(x=30, y=20)$, security capacity is about 0.8218 bit/symbol.

Wyner's theory on security capacity has proved that when security capacity of one region is greater than zero (that is, $\mathrm{Cs}>0$ ), in this region there must be some kind of communication method which could be absolute security theoretically. That is to say any eavesdropper in the region is unable to implement effective eavesdropping. We define this kind of region as a security area.

Similarly, when security capacity of one region less than zero (that is, $\mathrm{Cs}<0$ ), in this region there must be not any kind of communication method which could be absolute security theoretically. That is to say some eavesdropper in this region is able to implement effective eavesdropping. We define this kind of region as a no security region.

In this way, we can use security region and no security region to directly measure the size of region where theoretically can ensure the security communication in a communications system.

Obviously, with the same conditions, the larger the no security region is, the worse the physical layer security performance is; the smaller the no security region is, the better the physical layer security performance is.

In [7], it has changed PLNC location by using bit error rate (BER) and concluded that physical layer security performance of the system against passive eavesdropping is closely linked to PLNC node location.

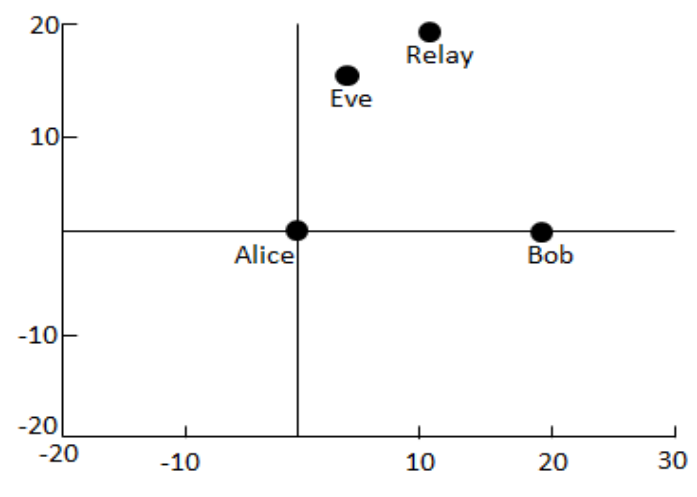

Figure 7. $\mathrm{R}(10,20)$ spatial locations.

Here we change the location of relay node between sender and receiver, then study on the security performance of PLNC. We assume the location of relay node $\mathrm{R}$ is $(0,10)$, $(0,20),(10,0),(10,20),(20,10)$, and $(20,20)$ respectively, which is shown in Fig.7.

In order to facilitate the observation, top view is adopted 


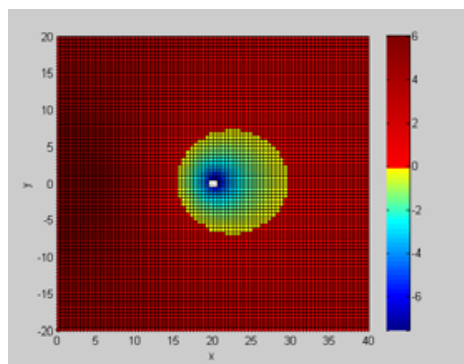

(a)

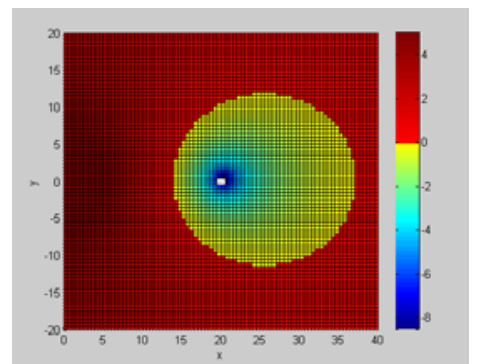

(b)

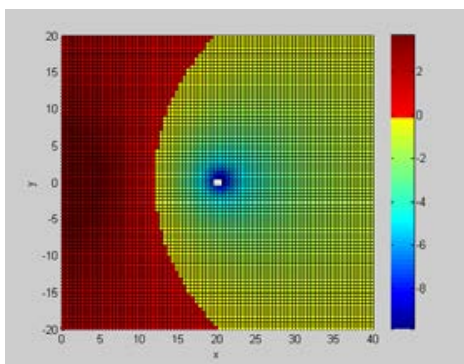

(c)

Figure 8. The top view of security capacity of A, when R is at the location of (a) $(10,0)$; (b) $(10,10)$; (c) $(10,20)$, in physical layer network coding mode.

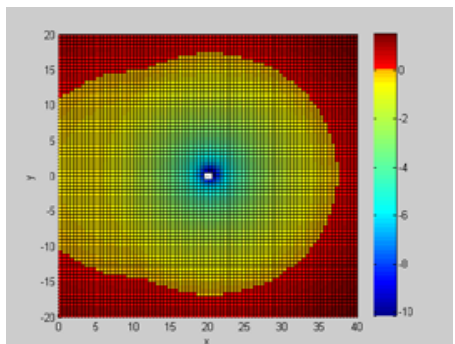

(a)

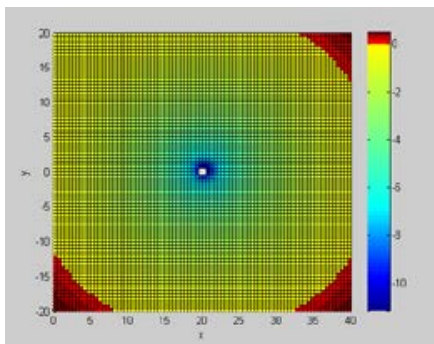

(b)

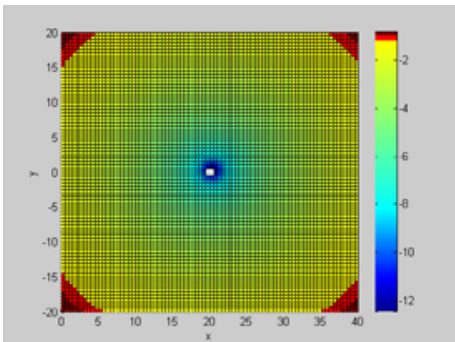

(c)

Figure 9. The top view of security capacity of A, when R is at the location of (a) $(10,0)$; (b) $(10,10)$; (c) $(10,20)$, in traditional wireless relay communication system.

Fig.8(a)(b)(c) is the top view of security capacity of A when $R$ is at the location of $(10,0),(10,10),(10,20)$ in physical layer network coding mode. In order to facilitate comparison, the top view of security capacity of A when R is at the location of $(10,0),(10,10),(10,20)$ in traditional wireless relay communication system is provided as Fig.9(a)(b)(c). The red color in Figure refers to security region (Cs>0). Comparing with Fig.8 and Fig.9, it is evident that when relay $\mathrm{R}$ has a longer distance from transmitter B, the no security region is larger and larger, which means the security performance is worse and worse. Therefore, from the perspective of physical layer security, we should make the relay node $\mathrm{R}$ closer to transmitter $\mathrm{B}$ as possible, so as to obtain a bigger security region.

In addition, compared with traditional wireless relay communication system, when the location of relay node remains the same, the size of security region of relay communication system based on PLNC increases significantly, which shows that physical layer network coding technology can improve the security performance of physical layer of wireless relay communication system.

\section{CONCLUSIONS}

This paper studies three-node wireless relay communication system based on PLNC, and provides theoretical expression of security capacity in the condition of unknown information about wiretap channel. In accordance with theoretical analysis and simulation results, compared with the traditional wireless relay communication system, either with high SNR and low SNR, PLNC technology can improve the physical layer security performance of wireless relay communication system.

In addition, when the eavesdropping position has a longer distance to the transmitter but a shorter distance to the receiver, its physical layer security performance is better. When relay $\mathrm{R}$ has a longer distance to the sender, the unsafe area is larger, and the safety performance is worse.

\section{REFERENCES}

[1] A.D.Wyner. The Wire-tap Channel [J]. Bell System Technical Journal, 1975, 54(8):1355-1387.

[2] S. Zhang, S. C. Liew, and P. P. Lam, Physical-layer network coding $[C]$, in Proc.Int.Conf.on Mobile Computing and Networking (MOBICOM), Los Angeles, CA, Sept. 2006, pp. 358-365.

[3] BinShi, LiangJin, ZhouZhong, JiangJi, To Improve Security Performance of Physical Layer of Wireless Relay System by Network Coding, Journal of Information Engineering University, 2011 No. 06.

[4] T.M .Cover and A .A .E .Carnal, Capacity Theorems for the Relay Channel[J], IEEE Trans.Info.Theory, vol.25 ,no5, Sept. 1979:572-584.

[5] YuehongShen,YuanyuanGao, YiminWei, Communication Theory[m], Mechanical Industry Publishing, Beijing, Second Edition, September 2008.

[6] YuanFeng, XiaoyunHou, etc.The Influence of Node Location on Security Capacity of Wiretap Channel, Development of Computer Technology December 2014, 12th, 24.

[7] M Hay, B Saeed,CH Lung,A Srinivasan.Co-Located Physical-Layer Network Coding to Mitigate Passive Eavesdropping, International Conference on Privacy Security \&Trust, 2010-1-2. 